as to whether HSV-2 serologic testing among asymptomatic persons results in persistent negative psychosocial consequences.

Methods Eight electronic databases and unpublished data sources were searched to identify studies measuring the psychosocial impact of HSV-2 serologic testing in persons without a history of genital herpes. To be included, studies had to test for HSV-2 using an HSV type-specific serologic test and to perform at least one psychosocial assessment of participants after they received HSV serologic results. We compared psychosocial responses in HSV-2 positive persons over time and vsHSV-2 negative persons (when available).

Results Nine studies satisfied the inclusion criteria. Studies were published from the years 2000-2008 and were conducted in the USA ( $N=6)$, Australia $(\mathrm{N}=2)$, and the UK $(\mathrm{N}=1)$. In total, 1355 participants were included; 596 (44\%) participants were HSV-2 positive, and of these 341 (57\%) lacked prior history of genital herpes. Participants were recruited from a variety of settings (ie, STD clinics, HMO enrollees, college campuses). Follow-up ranged from immediately after diagnosis to 1 year afterwards. Seven studies reported that HSV-2 diagnosis by serologic test did not have a persistent negative impact on participants' mental health (anxiety, depression, self-esteem) or sexual attitude and satisfaction. Two studies reported a negative impact of testing; one found that $5 \mathrm{HSV}$ 2 seropositive college students had increased distress 3 months posttesting as compared to HSV-2 negatives, and the other found selfreports of sexual undesirability up to one year after diagnosis. A genital herpes diagnosis was perceived as moderately severe for participants prior to testing; however, after HSV-2 testing, the perceived severity of a herpes diagnosis was lower among those testing HSV-2 positive.

Conclusions Diagnosis of HSV-2 by type-specific serologic testing did not result in long-term psychosocial harm in most asymptomatic persons. Concerns about sustained emotional impact should not deter clinicians from testing individuals without a history of genital herpes for HSV-2.

\section{P2-S3.11 STI-RELATED RISK BEHAVIOURS AND STI DISPARITY BETWEEN RESIDENTS OF BALTIMORE CITY AND OTHER URBAN CITIES IN THE USA}

doi:10.1136/sextrans-2011-050108.330

${ }^{1} \mathrm{M}$ Villarroel, ${ }^{2} \mathrm{~S}$ Rogers, ${ }^{3} \mathrm{C}$ Turner. ${ }^{1} \mathrm{~J}$ ohns Hopkins University, Baltimore, USA; ${ }^{2} \mathrm{RTI}$ International, Washington, District of Columbia, USA; ${ }^{3}$ City University of New York, Queens College and the Graduate Center, New York, USA

Background Baltimore City, Maryland, has experienced rates of STDs that are consistently higher than the national average. National surveillance data indicate that in 2000 the rates of gonorrhoea and Chlamydia in Baltimore City were 3.3 and 6.7 times higher than the overall US rate. Among US cities with greater than 200000 people, Baltimore City ranked among the top four for Chlamydia and gonorrhoea infection rates.

Objective To compare reports of diagnoses of gonorrhoea and Chlamydia among adults residing in Baltimore City to those in other central cities of the US and to assess whether a higher prevalence of sexual and substance use behaviours in Baltimore may account for infection disparity.

Methods We utilised data collected from a cross-sectional probability telephone survey of the USA $(\mathrm{N}=1,543)$ and Baltimore City $(\mathrm{N}=744)$ adults aged $18-45$ years old in 1999-2000. Respondents were asked about a wide range of STI-related risk behaviours and STI history. Bivariate analysis assessed differences in the prevalence of self-reported history of gonorrhoea and chlamydia, substance use, and sexual risk behaviours among residents of Baltimore City and other central cities of the USA Multivariate logistic regression models measured heterogeneity in self-reported history of gonorrhoea and chlamydia by location of residence, substance use and sexual history, adjusting for race and age.

Results Lifetime prevalence of gonorrhoea and chlamydia was $18.2 \%$ (95\% CI $14.8 \%$ to $22.1 \%$ ) among Baltimore residents and 9.8\% (95\% CI $7.3 \%$ to $13 \%$ ) among residents of other central cities ( $p<0.001)$. In bivariate analysis, Baltimore residents were no more likely to report a history of cocaine or injection drug use than residents of other urban areas $(p=0.774)$. However, Baltimore residents were more likely to report having six or more lifetime sexual partners (49.1\% vs $40.7 \%$, Prev. Ratio 1.21 (95\% CI 1.2 to 1.24$)$ ), multiple partners in the past year $(24.3 \%$ vs $16.9 \%$, Prev. Ratio 1.4 (95\% CI 1.4 to 1.5$)$ ), and a history of paid sex (17.8\% vs $8.7 \%$, Prev. Ratio 2.0 (95\% CI 1.8 to 2.3$)$ ).

Conclusion The higher prevalence of sexual risk behaviours among Baltimore adults is likely to accelerate STI transmission and contribute to the higher incidence of STIs in Baltimore.

\section{P2-S3.12 CULTURE AND RESEARCH: HOW DO YOU MIX THEM?}

doi:10.1136/sextrans-2011-050108.331

${ }^{1} \mathrm{D}$ Gesink, ${ }^{2} \mathrm{P}$ McGilvery, ${ }^{3} \mathrm{~T}$ Tilley, ${ }^{3} \mathrm{~K}$ Saganiuk. ${ }^{1}$ University of Toronto, Toronto, Canada; ${ }^{2}$ Saddle Lake Health Center, Saddle Lake, Canada; ${ }^{3}$ First Nations and Inuit Health, Health Canada, Saddle Lake, Canada

Background Community-based participatory research principles partner community and academics through all stages of the research process. Our purpose is to describe how we have been combining traditional Cree cultural practices (culture) and Western academic research processes (research) to facilitate a restorative research experience.

Methods Culture and research were given equal emphasis in the structure and content of meetings, governance structure of the project, knowledge exchange activities, project development and ethics. To begin this sexual health project, the Health Director invited academic and government partners to the community. Statistics on rates of sexually transmitted infections were shared with community partners. Since then, emphasis has been placed on face-to-face meetings in the community. Elders and community leadership have attended meetings and provided guidance on research activities. The governance structure was organised so primary decision makers are from the community and are guided by a community working group, cultural advisor and scientific advisor. Knowledge exchange was achieved through mutual participation in traditional Cree cultural activities and research emersion at an academic institution. Key community informants, Elders and research assistants led project development. The local First Nations College provided primary ethical review.

Results Face-to-face meetings helped build strong, sustainable relationships between community and outside researchers. Knowledge exchange activities, like cultural teachings and research trainings, contributed to mutual respect, understanding and trust between community and outside team members. Participation in community events and traditional ceremonies combined with formal and informal discussions with the community working group, Elders, and key community informants led to the identification of a priority research area of importance to the community. Following community led ways of knowing and doing has led to the development of innovative research methods for data collection. Ethics review held in ceremony provided a supportive, holistic environment from which to proceed with research activities.

Conclusions Building trust and nurturing the relationship between community and outside research partners has been integral for restoring confidence in the potential benefits of research for this First Nations community. 